\title{
A brand (new) experience: art, aesthetics, and sensory effects
}

\author{
Henrik Hagtvedt ${ }^{1}$ \\ Published online: 23 January 2022 \\ (c) Academy of Marketing Science 2021
}

Whereas marketers have historically focused on product functionality, experiential factors have become a primary basis for differentiation. Tesla was not the first functioning electric vehicle, but the sleek machine makes transportation exciting. Plenty of smartphones predated iPhones, but Apple brings wonder and delight to every stage of the user experience. Much of this experience is rooted in ancient sensory effects from our evolutionary past, but marketers make it new. In an effective blend of the familiar and the novel, modern experiential branding evokes the allure of sensory perception. If that perception is sufficiently interesting, pleasant, meaningful, or emotionally stirring, then it has aesthetic impact. Products and brands suffused by that aesthetic impact transcend the realm of functionality and enter the realm of art.

This editorial aims to highlight some insights and promising areas of research pertaining to these interrelated topics. Art, aesthetics, and sensory effects engage consumers and drive marketing performance, yet scholarship is lagging behind the marketplace in moving these topics from the fringe to the mainstream. At the same time, I want to emphasize how the topics hang together: Art is a focus area within aesthetics, and aesthetics is a focus area within sensory research; this perspective may aid in conceptually organizing findings and insights in these realms.

\section{Ancient roots and modern fruits}

Sensory perception is the primal basis for understanding the world, yet much remains to understand about the senses (Krishna, 2012). Marketers often appeal to consumers via explicit messages, perhaps relying on the logic of rational minds. Even peripheral cues that nudge consumers typically

Henrik Hagtvedt

hhagtvedt@gmail.com

1 Carroll School of Management, Boston College, Chestnut Hill, MA, USA convey a simple message. But one of the most fascinating aspects of sensory marketing is that it can invite exploration, thereby tingeing information with the joy of discovery. Not only do people tend to trust their own senses, but they also appreciate opportunities to interpret sensory input; people tend to enjoy that which has been evolutionarily adaptive, and a propensity to learn via sensory stimuli is a case in point. When marketers appeal directly to consumers' senses rather than provide explicit or thinly veiled messages, they can tap into primal mechanisms with an enduring potential for fresh, new experiences. These experiences may include diverse emotional components, ranging from the fear and disgust of horror movies to the enchantment of a welldesigned retail ambience.

Sensory influences can also slip under the radar when consumers are unaware. For instance, someone purchasing a vehicle may not realize how lighter or darker car paint affects perceptions of durability and user-friendliness. Similarly, the customer may not consider the psychoacoustic influence of the shutting car door on perceptions of sturdiness and quality, despite the amount of money and effort that car manufacturers expend on engineering just the right thud.

Perhaps unsurprisingly, vision is the sense that researchers have given most attention; it is generally considered the dominant sense, it recruits more of the brain than any of the other senses do, and it is relevant in most marketing contexts (Sample et al., 2020). Visual components such as shape and color play a central role in most design contexts, and scholars and brand managers alike recognize their importance.

However, the other senses represent fertile ground for investigation, too. Among them, touch is arguably most evidently primal, both in evolutionary terms and in terms of individual human lives. It is the first sense to develop in the womb and the last to vanish with old age. The need for touch is apparent in contexts ranging from a mother's embrace to the handling of objects in nature and products in the marketplace. Because consumers cannot physically handle products when shopping online, however, the current prevalence of digital screens offers both challenges and opportunities. For instance, marketers might attempt 
to simulate touch, or they can compensate for its absence with other sensory stimuli.

Similarly, consumers are unable to taste food displayed on a screen. But food is a multisensory experience. When we see and hear the sizzling steak, we can almost taste it with our mind's tongue. Relatedly, disconnects between our evolved preferences and current offerings present challenges for marketers and consumers. For example, our evolved desire for sweetness is presumably linked to the nutrients and energy provided by sweet fruits. However, sweet food is now in abundant supply, and it is much sweeter than anything that our ancestors could find in nature. The stage is thus set for conflicts between health and cravings.

Whereas touch and taste require proximity, the auditory and olfactory senses are similar to vision in that they can operate across a greater distance. Future research may expand on the current knowledge of tools that marketers already use, such as sonic logos or ambient music and scent, or it may uncover new tools or new uses for existing ones. Notably, technological changes tend to spawn new opportunities. For instance, digital scent technology stretches back to the late 1950s, when movie theaters began including odor emissions. By the new millennium, marketers introduced peripheral computer devices designed to emit odors when users opened a web site or email. Today, a scent device can be attached to AR or VR headsets. One of the hurdles in these developments is the need to better understand olfactory perception. Relatedly, all the senses are likely to play a role in any future development of the metaverse, a collective space combining virtual and augmented reality technologies in an online realm.

A great deal of research is needed to illuminate the human sensorium in general, whether in terms of individual senses or crossmodal correspondences and interactions. In particular, research should shed light on characteristic aspects of sensory communication such as exploration, interpretation, and emotional responses, thereby also establishing advantages and disadvantages compared with other types of messaging. Whether sensory signals are concrete (e.g., an aroma conveying a specific flavor) or abstract (e.g., a visual design conveying brand personality), they invite consumers to discover and interpret information, which provides both opportunities and potential pitfalls for marketers. Additionally, this topic provides unique opportunities to study current behaviors with easily identifiable primal roots, which can aid in elucidating cultural differences among universal tendencies. Sensory effects may combine universal aspects and idiosyncratic ones based on culture or technological innovations, and the basis for these effects may range from simple, barely detectable stimuli to constellations of input that kindle aesthetic interest.

\section{Prevalence of aesthetics}

From a research standpoint, aesthetics represents a sub-section of the broader field of sensory experience. The study of aesthetics initially developed as theories of art appreciation, but it has long since expanded to include any perceivable object, and most marketing research in this domain focuses on ordinary consumer products. Much of this work revolves around aesthetic appeal and related general assessments, whereas other work focuses on specific aesthetic principles such as complexity, novelty, symmetry, balance, unity, contrast, and fluency.

When conceptualizing aesthetics, some scholars focus narrowly on beauty, whereas others focus more broadly on sensory effects. The former arguably reduces the construct to a redundant synonym, whereas the latter can blur the boundary between aesthetics and general sensory perception. In my view, the most useful conceptualization lies somewhere in the middle: Aesthetics arises from sensory perception in any modality, but it is restricted to experience that is interesting, pleasant, meaningful, or stirring, often with beauty as a central component.

Aesthetics is a growing area of research in marketing, although it does not yet receive as much attention as it merits based on its role in the marketplace. Aesthetic design has become a key differentiator between brands and a potent source of product appeal, and not just for high-end products such as the aforementioned Teslas and iPhones. It is pervasive in the marketplace, and even retailers like Target collaborate with designers to offer collections with signature aesthetic appeal.

Consistent with the aforementioned conceptualization of aesthetics, research in this area should not be restricted to perceptions of beauty; it should map out various aspects of sensory experience that involve interest, pleasure, meaning, or emotional responses. Within each of these sub-topics, there is a wide range of opportunities for future investigation. For instance, whereas aesthetic designs can convey meaning by signaling specific product- or brand characteristics, they might also influence consumers' perceptions of meaningful brand relationships, or even overall meaning in life. Further, marketing-relevant effects can arise within a wide range of contexts, including mundane ones for which aesthetics are not an obvious consideration. For example, a well-designed pricing screen may be pleasantly easy to read, thereby facilitating sales, whether or not the design is particularly creative.

\section{The art of employing art}

On the other hand, when aesthetic design reflects ample creativity and skill, it approaches the realm of art. Much as aesthetics represents a sub-section of the broader field of 
sensory experience, art represents a focus area within the broader field of aesthetics. In turn, research on art comprises at least three promising sub-areas: arts marketing (Colbert \& St-James, 2014), the psychological experience of art (Hagtvedt $\&$ Vohs, 2021), and the use of art in the marketing of other products (Hagtvedt \& Patrick, 2008). Notably, the phenomenon of art infusion pertains to the influence of artworks on consumer responses to objects (e.g., products, brands) with which the artworks are associated. The link to artworks can arise in various ways, including advertising, labeling, product- and packaging design, sponsorship, or mere physical proximity. As long as there is a perceived association, perceptions can spill over from artworks to non-art objects, thereby encouraging favorable consumer responses. Prior work has demonstrated the role of luxury perceptions in such spillovers, but future work may uncover additional mechanisms. Further, research in this area should move beyond general evaluations to more nuanced or specific measures.

Every society on historical record has engaged in art; this mode of communication clearly has an extremely widespread and enduring appeal, from which marketers can benefit. In particular, the ambiguity of artworks may be instrumental in sparking curiosity and engaging consumers' interest. Art resides in the manner in which something is expressed, not so much in what, specifically, is being expressed. Art therefore differs starkly from most marketing messages, which typically aim to persuade. Consider a painting of a shoebox, a random face, an abstract sensation, or anything else depicted on a canvas. The subject matter (i.e., the literal information being conveyed) is not what makes it art; it is the manner of depiction that matters. Interplays between sensory building blocks such as colors, shapes, rhythms, sounds, and movements need not convey specific or literal information, but they contribute to aesthetic impact. Depending on how one defines art, these interplays can also include conceptual building blocks, but if so, it is the audience's prerogative to interpret their significance.

This type of ambiguous communication has its risks, and it may not befit all marketing contexts. However, it has a unique capacity to generate interest. And that interest can be cultivated; consumers appreciate the sensory interplays of art to varying degrees, but the appreciation tends to increase with experience. Jazz improvisations can seem like a bewildering cacophony to the uninitiated, whereas a more practiced listener might enjoy complex chord interactions and creative deviations from expectation. Similarly, poetry, painting, sculpture, and dance invite interpretation and mental exploration, which can evolve over time. In other words, art is a renewable source of interest and meaning, some of which transfers to products or brands infused with that source. Future research should investigate the impacts of different kinds of art and artistic components, the process whereby different meanings emerge from those artworks, the characteristics of products and brands that lend themselves to integration with art, and the consumer characteristics that influence both art appreciation and responses to interplays between art and marketing. Consistent with this latter notion, and similar to the aforementioned observations regarding aesthetics and general sensory effects, research on art lends itself to explicating cultural and individual variety within universal tendencies.

\section{Nature, nurture, culture, and future}

On that note, I will conclude with some additional thoughts about innate and learned aspects of meaningful sensory experiences. Understanding both is particularly useful in an increasingly multicultural marketplace, especially given the central roles of variables such as tradition and individual tastes in these domains.

With respect to culturally learned aspects, a marketer might target a given segment by including art or aesthetic design elements with which those consumers identify, or incorporate familiar sounds, scents, or other sensory stimuli. This kind of sensory communication can trigger recognition and belonging, although it may require that marketers maintain a perception of authenticity, lest they risk alienating the consumers they are trying to attract. At the same time, such efforts can represent learning opportunities for consumers from other cultures. Relatedly, appreciating art often entails empathizing with the people and cultures from which the artworks spring. In philosophy of art, in fact, there is a longstanding notion that empathy is a key component of aesthetic experience. Marketers can draw on this insight to the benefit of business goals as well as consumer wellbeing; the use of art can encourage multiculturalism and tolerance, while promoting consumer products to diverse markets.

Marketers can also attempt broader outreach by relying on universal aspects of sensory experience, which presumably stem from a common evolutionary heritage. I have long held the theory that aesthetic appreciation must predate spoken language, and probably by a lot. Even prehominid ancestors must have interpreted the environment based on sensory perception, and the tendency for sensory input with favorable connotations to stimulate pleasure or interest - the beginnings of aesthetic appreciation-presumably spread as progenitors drawn to the related advantages tended to survive, thrive, and ultimately propagate their genes. It does not take a great leap of imagination to see that at some point, humans would also begin to manipulate and exaggerate such sensory stimuli, thereby creating art. This heritage, too, suggests that aesthetics can bridge cultural divides; if the creation and appreciation of art has deep roots in human nature, then intercultural variety in art presumably does not preclude connection and understanding between those 
cultures. Because aesthetics is a common language, art may extend a brand's reach while simultaneously contributing to the growth of empathy between diverse communities.

Future research may deepen our understanding of this language, whether at the universal, culture-specific, or individual level. Not only can that research elucidate the broad appeal of existing products such as Teslas and iPhones, but it also may pave the way for future innovations. The topics of art, aesthetics, and sensory effects invite creativity from scholars; rather than lagging behind the marketplace, research on these topics should also aid its future evolution.

\section{References}

Colbert, F., \& St-James, Y. (2014). Research in arts marketing: Evolution and future directions. Psychology \& Marketing, 31(8), $566-575$
Hagtvedt, H., \& Patrick, V. M. (2008). Art infusion: The influence of visual art on the perception and evaluation of consumer products. Journal of Marketing Research, 45(3), 379-389.

Hagtvedt, H., \& Vohs, K. D. (2021). Viewing challenging art lends meaning to life by stimulating integrative complexity. The Journal of Positive Psychology. https://doi.org/10.1080/17439760.2021. 1975159. forthcoming.

Krishna, A. (2012). An integrative review of sensory marketing: Engaging the senses to affect perception, judgment, and behavior. Journal of Consumer Psychology, 22(3), 332-351.

Sample, K. L., Hagtvedt, H., \& Brasel, S. A. (2020). Components of visual perception in marketing contexts: A conceptual framework and review. Journal of the Academy of Marketing Science, 48(3), $405-421$.

Publisher's note Springer Nature remains neutral with regard to jurisdictional claims in published maps and institutional affiliations. 